\title{
The Quantum Description of BF Model in Superspace
}

\author{
Manoj Kumar Dwivedi iD \\ Department of Physics, Banaras Hindu University, Varanasi 221005, India \\ Correspondence should be addressed to Manoj Kumar Dwivedi; manojdwivedi84@gmail.com
}

Received 29 May 2017; Revised 8 August 2017; Accepted 10 August 2017; Published 10 January 2018

Academic Editor: Elias C. Vagenas

Copyright (C) 2018 Manoj Kumar Dwivedi. This is an open access article distributed under the Creative Commons Attribution License, which permits unrestricted use, distribution, and reproduction in any medium, provided the original work is properly cited. The publication of this article was funded by SCOAP ${ }^{3}$

We consider the BRST symmetric four-dimensional BF theory, a topological theory, containing antisymmetric tensor fields in Landau gauge and extend the BRST symmetry by introducing a shift symmetry to it. Within this formulation, the antighost fields corresponding to shift symmetry coincide with antifields of standard field/antifield formulation. Furthermore, we provide a superspace description for the BF model possessing extended BRST and extended anti-BRST transformations.

\section{Introduction}

Topological gauge field theories (TGFT) which came from mathematics have some peculiar features. The examples of two distinct class of TGFT are topological Yang-Mills theory and Chern-Simons (CS) theory, which are sometimes classified as Witten-type and Schwarz-type, respectively [1]. Besides these two types, there is another Schwarz-type TGFT called topological BF theory, which is an extension of CS theory [2]. The difference between CS theory and BF model is that action of previous theory exists only in odddimensions while later one can be defined on manifolds of any dimensions.

In string theory and nonlinear sigma model, fourdimensional antisymmetric (or BF) models [3-8] were introduced some years ago. This model is interesting due to its topological nature [1] and its connection with lower dimensional quantum gravity; for example, three space-time dimensional Einstein-Hilbert models with or without using cosmological constant can be naturally formulated in terms of BF-models $[9,10]$. Coupling of an antisymmetric tensor field with the field strength tensor of Yang-Mills is described by these models [11]. Quantization of BF model in Landau gauge has been studied in [11]. Topological BF theory in Landau gauge has a common feature of a large class of topological models [12-16].

On the other hand, the Batalin-Vilkovisky (BV) approach, also known as field/antifield formulation, [17-20] is one of the most powerful quantization algorithms presently available. BV formulation deals with very general gauge theories, including those with open or reducible gauge symmetry algebras. The BV method also address the possible violations of symmetries of the action by quantum effects. The BV formulation (independently introduced by Zinn-Justin [21]) extends the BRST approach [22-36]. In fact, the BRST symmetry $[37,38]$ is a very important symmetry for gauge theories [26,39-54]. Besides the covariant description to perform the gauge-fixing in quantum field theory, BV formulation was also applied to other problems like analysing possible deformations of the action and anomalies.

A superspace description for various gauge theories in BV formulation has been studied extensively [55-64]. They have shown that the extended BRST and extended antiBRST invariant actions of these theories (including some shift symmetry) in BV formulation yield naturally the proper identification of the antifields through equations of motion. The shift symmetry is important and gets relevance, for example, in inflation particularly in supergravity [65] as well as in Standard Model [66]. In usual BV formulation, these antifields can be calculated from the expression of gauge-fixing fermion. We extended BRST formulation and superspace description of the topological gauge (BF) model is still unstudied and we try to discuss these here.

In the present work, we try to generalize the superspace formulation of BV action for BF model. Particularly, we first consider BRST invariant BF model in Landau gauge 
and extend the BRST symmetry of the theory by including shift symmetry. By doing so, we find that the antighosts of shift symmetry get identified as antifields of standard BV formulation naturally. Further, we discuss a superspace formulation of extended BRST invariant BF model. Here we see that one additional Grassmann coordinate is required if action admits only extended BRST symmetry. However, for both extended BRST and extended anti-BRST invariant BF model two additional Grassmann coordinates are required.

This paper is framed as follows. In Section 2, we discuss the BRST invariant BF model. In Section 3, we study the extended BRST transformation of the model. Further, we describe extended BRST invariant action in superspace in Section 4 . The extended anti-BRST symmetry is discussed in Section 5. The superspace formulation of extended BRST and anti-BRST invariant action is given in Section 6. The last section is reserved for concluding remarks.

\section{BRST Invariant BF Model}

In this section, we discuss the preliminaries of $\mathrm{BF}$ model with its BRST invariance. In this view, the BF model in flat $(3+1)$ space-time dimensions is given by the following gauge invariant Lagrangian density [11]:

$$
\mathscr{L}_{0}=-\frac{1}{4} \epsilon^{\mu \nu \rho \sigma} F_{\mu \nu}^{a} B_{\rho \sigma}^{a}
$$

where $B_{\rho \sigma}^{a}$ and $F_{\mu \nu}^{a}$ are two-form field and field strength tensor for vector field, respectively. In order to remove discrepancy due to gauge symmetry, the gauge-fixing and ghost terms are given by

$$
\begin{aligned}
& \mathscr{L}_{g f+g h} \\
& =b^{a} \partial^{\mu} A_{\mu}^{a}+\bar{C}^{a} \partial^{\mu}\left(D_{\mu} C\right)^{a}+h^{a \nu}\left(\partial^{\mu} B_{\mu \nu}^{a}\right)+\omega^{a} \partial \xi^{a} \\
& +h_{\mu}^{a}\left(\partial^{\mu} e^{a}\right)+\omega^{a} \lambda^{a}+\left(\partial^{\mu} \bar{\xi}_{\mu}^{a}\right) \lambda^{a} \\
& \quad-\left(\partial^{\mu} \bar{\phi}^{a}\right)\left[\left(D_{\mu} \phi\right)^{a}+f^{a b c} c^{b} \xi_{\mu}^{c}\right] \\
& \quad-\left(\partial^{\mu} \bar{\xi}^{a \nu}\right)\left[\left(D_{\mu} \xi_{\nu}\right)^{a}-\left(D_{\nu} \xi_{\mu}\right)^{a}+f^{a b c} B_{\mu \nu}^{b} C^{c}\right] \\
& +\frac{1}{2} f^{a b c} \epsilon^{\mu \nu \rho \sigma}\left(\partial_{\mu} \bar{\xi}_{\nu}^{a}\right)\left(\partial_{\rho} \bar{\xi}_{\sigma}^{b}\right) \phi^{c},
\end{aligned}
$$

where fields $\left(C^{a}, \xi_{\mu}^{a}\right),\left(\bar{C}^{a}, \bar{\xi}_{\mu}^{a}\right)$, and $\left(b^{a}, h_{\mu}^{a}\right)$ are the ghosts, antighosts, and the multipliers fields, respectively, while the fields $\phi^{a}, \bar{\phi}^{a}$ and $\omega^{a}$ are taken into account to remove further degeneracy due to the existence of zero modes in the transformations.
The effective Lagrangian density of BF model, $\mathscr{L}=\mathscr{L}_{0}+$ $\mathscr{L}_{g f+g h}$, possesses the following BRST symmetry:

$$
\begin{aligned}
s A_{\mu}^{a}= & -\left(D_{\mu} C\right)^{a}, \\
s C^{a}= & \frac{1}{2} f^{a b c} C^{b} C^{c}, \\
s \xi_{\mu}^{a}= & \left(D_{\mu} \phi\right)^{a}+f^{a b c} C^{b} \xi_{\mu}^{c}, \\
s B_{\mu \nu}^{a}= & -\left(D_{\mu} \xi_{\nu}-D_{\nu} \xi_{\mu}\right)^{a}-f^{a b c} B_{\mu \nu}^{b} C^{c} \\
& +f^{a b c} \epsilon_{\mu \nu \rho \sigma}\left(\partial^{\rho} \bar{\xi}^{b \sigma}\right) \phi^{c}, \\
s \phi^{a}= & f^{a b c} C^{b} \phi^{c}, \\
s \bar{\xi}_{\mu}^{a}= & h_{\mu}^{a}, \\
s \bar{C}^{a}= & b^{a}, \\
s \bar{\phi}^{a}= & \omega^{a}, \\
s e^{a}= & \lambda^{a}, \\
s\left(h_{\mu}^{a}, b^{a}, \omega \omega^{a}, \lambda^{a}\right)= & 0 .
\end{aligned}
$$

The gauge-fixing and ghost terms of the effective Lagrangian density are BRST exact and, hence, can be written in terms of BRST variation of gauge-fixing fermion,

$$
=\left(\bar{C}^{a} \partial^{\mu} A_{\mu}^{a}+\bar{\xi}^{a \mu} \partial^{\nu} B_{\mu \nu}^{a}+\bar{\phi}^{a} \partial^{\mu} \xi_{\mu}^{a}-e^{a} \omega^{a}-e^{a} \partial^{\mu} \bar{\xi}_{\mu}^{a}\right),
$$

as follows:

$$
\mathscr{L}_{g f+g h}=s \Psi .
$$

In the next section, we would like to study the extended BRST symmetry for the model which incorporates shift symmetry together with original BRST symmetry.

\section{Extended BRST Invariant Lagrangian Density}

The advantage of studying the extended BRST transformations for BF model in BV formulation is that antifields get identification naturally. We begin with shifting all the fields from their original value as follows:

$$
\begin{aligned}
& B_{\mu \nu}^{a} \longrightarrow B_{\mu \nu}^{a}-\widetilde{B}_{\mu \nu}^{a}, \\
& A_{\mu}^{a} \longrightarrow A_{\mu}^{a}-\widetilde{A}_{\mu}^{a}, \\
& C^{a} \longrightarrow C^{a}-\widetilde{C}^{a}, \\
& \bar{C}^{a} \longrightarrow \bar{C}^{a}-\widetilde{\bar{C}}^{a}, \\
& b^{a} \longrightarrow b^{a}-\widetilde{b}^{a},
\end{aligned}
$$




$$
\begin{gathered}
\xi_{\mu}^{a} \longrightarrow \xi_{\mu}^{a}-\tilde{\xi}_{\mu}^{a}, \\
\bar{\xi}_{\mu}^{a} \longrightarrow \bar{\xi}_{\mu}^{a}-\widetilde{\bar{\xi}}_{\mu}, \\
\phi^{a} \longrightarrow \phi^{a}-\tilde{\phi}^{a}, \\
\bar{\phi}^{a} \longrightarrow \bar{\phi}^{a}-\widetilde{\bar{\phi}}^{a}, \\
h_{\mu}^{a} \longrightarrow h_{\mu}^{a}-\widetilde{h}_{\mu}^{a}, \\
e^{a} \longrightarrow e^{a}-\widetilde{e}^{a}, \\
\omega^{a} \longrightarrow \omega^{a}-\widetilde{\omega}^{a}, \\
\lambda^{a} \longrightarrow \lambda^{a}-\widetilde{\lambda}^{a} .
\end{gathered}
$$

$$
\begin{aligned}
s \widetilde{e}^{a} & =N^{a}-\left(\lambda^{a}-\widetilde{\lambda}^{a}\right), \\
s \xi_{\mu}^{a} & =L_{\mu}^{a}, \\
s \bar{C}^{a} & =\bar{\epsilon}^{a}, \\
s \widetilde{\xi}_{\mu}^{a} & =L_{\mu}^{a}-\left[\left(D_{\mu}-\widetilde{D}_{\mu}\right)(\phi-\widetilde{\phi})^{a}\right. \\
& \left.+f^{a b c}\left(C^{b}-\widetilde{C}^{b}\right)\left(\xi_{\mu}^{c}-\widetilde{\xi}_{\mu}^{c}\right)\right], \\
s \bar{\xi}_{\mu}^{a} & =\bar{L}_{\mu}^{a}, \\
\widetilde{c}_{\xi_{\mu}^{a}} & =\bar{L}_{\mu}^{a}-\left(h_{\mu}^{a}-\widetilde{h}_{\mu}^{a}\right),
\end{aligned}
$$

(6)

The effective Lagrangian density of BF model also gets shifted under such shifting of fields, respectively. This is given by

$$
\begin{aligned}
\widetilde{\mathscr{L}} & =\mathscr{L}\left(A_{\mu}^{a}-\widetilde{A}_{\mu}^{a}, C^{a}-\widetilde{C}^{a}, \bar{C}^{a}-\widetilde{\bar{C}}^{a}, b^{a}-\widetilde{b}^{a}, \xi_{\mu}^{a}\right. \\
& -\widetilde{\xi}_{\mu}^{a}, \bar{\xi}_{\mu}^{a}-{\widetilde{\xi^{a}}}_{\mu}, \phi^{a}-\widetilde{\phi}^{a}, \bar{\phi}^{a}-\widetilde{\bar{\phi}}^{a}, h_{\mu}^{a}-\widetilde{h}_{\mu}^{a}, e^{a}-\widetilde{e}^{a}, \omega^{a} \\
& \left.-\widetilde{\omega}^{a}, \lambda^{a}-\widetilde{\lambda}^{a}\right) .
\end{aligned}
$$

The shifted Lagrangian density is invariant under BRST transformation together with a shift symmetry transformation, jointly known as extended BRST transformation. The extended BRST symmetry transformations under which Lagrangian density of BF model is invariant are written by

$$
\begin{aligned}
& s A_{\mu}^{a}=\psi_{\mu}^{a}, \\
& s \widetilde{A}_{\mu}^{a}=\psi_{\mu}^{a}-\left(D_{\mu}-\widetilde{D}_{\mu}\right)(C-\widetilde{C})^{a}, \\
& s C^{a}=\epsilon^{a}, \\
& s \widetilde{C}^{a}=\epsilon^{a}-\frac{1}{2} f^{a b c}\left(C^{b}-\widetilde{C}^{b}\right)\left(\xi_{\mu}^{a}-\widetilde{\xi}_{\mu}^{a}\right), \\
& \widetilde{\bar{C}}^{a}=\bar{\epsilon}^{a}-(b-\widetilde{b})^{a}, \\
& s b^{a}=\chi^{a}, \\
& s \widetilde{b}^{a}=\chi^{a}, \\
& s \phi^{a}=M^{a}, \\
& s \widetilde{\phi}^{a}=M^{a}-f^{a b c}\left(C^{b}-\widetilde{C}^{b}\right)\left(\phi^{c}-\widetilde{\phi}^{c}\right), \\
& s \bar{\phi}^{a}=\bar{M}^{a}, \\
& \widetilde{s \phi}=M^{a}-\left(\omega^{a}-\widetilde{\omega}^{a}\right), \\
& s e^{a}=N^{a},
\end{aligned}
$$
fields corresponding to shift symmetry for $A_{\mu}^{a}, C^{a}, \bar{C}^{a}, b^{a}$, $\phi^{a}, \bar{\phi}^{a}, e^{a}, \xi_{\mu}^{a}$ and $\bar{\xi}_{\mu}^{a}$, respectively. The nilpotency of extended BRST symmetry (8) leads to the BRST transformation for the following ghost fields:

$$
\begin{gathered}
s \psi_{\mu}^{a}=0, \\
s \epsilon^{a}=0, \\
s \bar{\epsilon}^{a}=0, \\
s \chi^{a}=0, \\
s M^{a}=0, \\
s \bar{M}^{a}=0, \\
s N^{a}=0, \\
s L_{\mu}^{a}=0, \\
s \bar{L}_{\mu}^{a}=0 .
\end{gathered}
$$

In order to make the theory ghost free, we need further antighosts $A_{\mu}^{\star a}, C^{\star a}, \bar{C}^{\star a}, b^{\star a}, \xi_{\mu}^{\star a}, \bar{\xi}_{\mu}^{\star a}, \phi^{\star a}, \bar{\phi}^{\star a}$, and $e^{\star a}$ to be introduced corresponding to the ghost fields $\psi_{\mu}^{a}, \epsilon^{a}, \bar{\epsilon}^{a}, \chi^{a}, M^{a}, \bar{M}^{a}, N^{a}, L_{\mu}^{a}$, and $\bar{L}_{\mu}^{a}$, respectively. The BRST transformations of these antighosts are constructed as follows:

$$
\begin{aligned}
s A_{\mu}^{\star a} & =-\zeta_{\mu}^{a}, \\
s C^{\star a} & =-\sigma^{a}, \\
s \bar{C}^{\star a} & =-\bar{\sigma}^{a}, \\
s b^{\star a} & =-\omega^{a}, \\
s \phi^{\star a} & =-v^{a}, \\
s \bar{\phi}^{\star a} & =-\bar{v}^{a},
\end{aligned}
$$

where $\psi_{\mu}^{a}, \epsilon^{a}, \bar{\epsilon}^{a}, \chi^{a}, M^{a}, \bar{M}^{a}, N^{a}, L_{\mu}^{a}$, and $\bar{L}_{\mu}^{a}$ are the ghost 


$$
\begin{aligned}
& s e^{\star a}=-\tau^{a}, \\
& s \xi_{\mu}^{\star a}=-\kappa^{a}, \\
& s \bar{\xi}_{\mu}^{\star a}=-\bar{\kappa}^{a},
\end{aligned}
$$

where $\zeta_{\mu}^{a}, \sigma^{a}, \bar{\sigma}^{a}, \omega^{a}, v^{a}, \tau^{a}, \kappa^{a}$, and $\bar{\kappa}^{a}$ are the NakanishiLautrup type auxiliary fields corresponding to shifted fields $\widetilde{A}_{\mu}^{a}, \widetilde{C}^{a}, \widetilde{\bar{C}}^{a}, \widetilde{b}^{a}, \widetilde{\phi}^{a}, \widetilde{\bar{\phi}}^{a}, \widetilde{e}^{a}, \widetilde{\xi}_{\mu}^{a}$, and ${\widetilde{\bar{\xi}^{a}}}_{\mu}$ having the following BRST transformations:

$$
\begin{aligned}
& s \zeta_{\mu}^{a}=0, \\
& s \sigma^{a}=0, \\
& s \bar{\sigma}^{a}=0, \\
& s{\omega^{a}}^{a}=0, \\
& s v^{a}=0, \\
& s \bar{v}^{a}=0, \\
& s \tau^{a}=0, \\
& s \kappa^{a}=0, \\
& s \bar{\kappa}^{a}=0 .
\end{aligned}
$$

We can recover our original BF model by fixing the shift symmetry in such a way such that effect of all the tilde fields will vanish. We achieve this by adding following gauge-fixed term to the shifted Lagrangian density (7):

$$
\begin{aligned}
& \widetilde{\mathscr{L}}_{g f+g h}=-\zeta^{a \mu} \widetilde{A}_{\mu}^{a}-A_{\mu}^{a \star}\left[\psi^{a \mu}-\left(D^{\mu}-\widetilde{D}^{\mu}\right)(C\right. \\
& \left.-\widetilde{C})^{a}\right]-\bar{\sigma}^{a} \widetilde{C}^{a}+\bar{C}^{a \star}\left[\epsilon^{a}-\frac{1}{2} f^{a b c}\left(C^{b}-\widetilde{C}^{b}\right)\left(\xi_{\mu}^{a}\right.\right. \\
& \left.\left.-\widetilde{\xi}_{\mu}^{a}\right)\right]-\sigma^{a} \widetilde{\bar{C}}^{a}+C^{a \star}\left[\bar{\epsilon}^{a}-\left(b^{a}-\widetilde{b}^{a}\right)\right]-v^{a} \widetilde{\phi}^{a} \\
& -\phi^{\star a}\left[M^{a}-f^{a b c}\left(C^{b}-\widetilde{C}^{b}\right)\left(\phi^{c}-\widetilde{\phi}^{c}\right)\right]-\bar{v}^{a} \widetilde{\bar{\phi}}^{a} \\
& -\bar{\phi}^{\star a}\left(\bar{M}^{a}-\omega^{a}+\widetilde{\omega}^{a}\right)-\tau^{a} \widetilde{e}^{a}-e^{\star}\left[N^{a}-\lambda^{a}+\widetilde{\lambda}^{a}\right] \\
& -\Phi^{a} \widetilde{b}^{a}-b^{\star a} \chi^{a}-\kappa^{a} \widetilde{\xi}_{\mu}^{a}-\bar{\kappa}^{a} \widetilde{\bar{\xi}}_{\mu}^{a}+\xi_{\mu}^{\star a}\left(L_{\mu}^{a}\right. \\
& -\left[( D _ { \mu } - \widetilde { D } _ { \mu } ) \left(\phi-\widetilde{\phi}^{a}\right.\right. \\
& \left.\left.+f^{a b c}\left(C^{b}-\widetilde{C}^{b}\right)\left(\xi_{\mu}^{c}-\widetilde{\xi}_{\mu}^{c}\right)\right]\right)+\bar{\xi}_{\mu}^{\star a}\left[\bar{L}_{\mu}^{a}-\left(h_{\mu}^{a}\right.\right. \\
& \left.\left.-\widetilde{h}_{\mu}^{a}\right)\right] .
\end{aligned}
$$

One can easily check that this gauge-fixing Lagrangian density $\widetilde{\mathscr{L}}_{g f+g h}$ also admits the extended BRST invariance.
Integrating the auxiliary fields of the above expression, we obtain

$$
\begin{aligned}
\widetilde{\mathscr{L}}_{g f+g h}= & -A_{\mu}^{a \star}\left[\psi^{a \mu}-\left(D^{\mu} C\right)^{a}\right] \\
& +\bar{C}^{a \star}\left[\epsilon^{a}-\frac{1}{2} f^{a b c} C^{b} \xi_{\mu}^{c}\right]+C^{a \star}\left[\bar{\epsilon}^{a}-b^{a}\right] \\
& -\phi^{\star a}\left(M^{a}-f^{a b c} C^{b} \phi^{c}\right)-\bar{\phi}^{\star a}\left(\bar{M}^{a}-\omega^{a}\right) \\
& -e^{\star a}\left[N^{a}-\lambda^{a}\right]-b^{\star a} \chi^{a} \\
& +\xi_{\mu}^{\star a}\left(L_{\mu}^{a}-\left[\left(D_{\mu} \phi\right)^{a}+f^{a b c} C^{b} \xi_{\mu}^{c}\right]\right) \\
& +\bar{\xi}_{\mu}^{\star a}\left[\bar{L}_{\mu}^{a}-h_{\mu}^{a}\right] .
\end{aligned}
$$

The gauge-fixing and ghost terms of the Lagrangian density are BRST exact and can be expressed in terms of a general gauge-fixing fermion $\Psi$ as

$$
\begin{aligned}
s \Psi= & s A_{\mu}^{a} \frac{\delta \Psi}{\delta A_{\mu}^{a}}+s C^{a} \frac{\delta \Psi}{\delta C^{a}}+s \bar{C}^{a} \frac{\delta \Psi}{\delta \bar{C}^{a}}+s b^{a} \frac{\delta \Psi}{\delta b^{a}} \\
& +s \xi_{\mu}^{a} \frac{\delta \Psi}{\delta \xi_{\mu}^{a}}+s \bar{\xi}_{\mu}^{a} \frac{\delta \Psi}{\delta \bar{\xi}_{\mu}^{a}}+s \phi^{a} \frac{\delta \Psi}{\delta \phi^{a}}+s \bar{\phi} \frac{\delta \Psi}{\delta \bar{\phi}^{a}} \\
& +s e^{a} \frac{\delta \Psi}{\delta e^{a}} \\
= & -\frac{\delta \Psi}{\delta A_{\mu}^{a}} \psi_{\mu}^{a}+\frac{\delta \Psi}{\delta C^{a}} \epsilon^{a}+\frac{\delta \Psi}{\delta \bar{C}^{a}} \bar{\epsilon}^{a}-\frac{\delta \Psi}{\delta b^{a}} \chi^{a}-\frac{\delta \Psi}{\delta \xi_{\mu}^{a}} L_{\mu}^{a} \\
& -\frac{\delta \Psi}{\delta \bar{\xi}_{\mu}^{a}} \bar{L}_{\mu}^{a}-\frac{\delta \Psi}{\delta \phi^{a}} M^{a}-\frac{\delta \Psi}{\delta \bar{\phi}^{a}} \bar{M}^{a}-\frac{\delta \Psi}{\delta e^{a}} N^{a} .
\end{aligned}
$$

After integrating out the auxiliary fields which set the tilde fields to zero, we have the complete effective action for BF model in Landau gauge possessing extended BRST symmetry as

$$
\begin{aligned}
\mathscr{L}_{\mathrm{eff}}= & \mathscr{L}_{0}+\mathscr{L}_{g f+g h}+\widetilde{\mathscr{L}}_{g f+g h} \\
= & \mathscr{L}_{0}+\left(-A_{\mu}^{\star a}-\frac{\delta \Psi}{\delta A^{\mu a}}\right) \psi^{\mu a} \\
& +\left(\bar{C}^{\star a}+\frac{\delta \Psi}{\delta C^{a}}\right) \epsilon^{a}+\left(C^{\star a}+\frac{\delta \Psi}{\delta \bar{C}^{a}}\right) \bar{\epsilon}^{a} \\
& -\left(b^{\star a}+\frac{\delta \Psi}{\delta b^{a}}\right) \chi^{a}+\left(\xi_{\mu}^{\star a}+\frac{\delta \Psi}{\delta \xi_{\mu}^{a}}\right) L_{\mu}^{a} \\
& +\left(\bar{\xi}_{\mu}^{\star a}+\frac{\delta \Psi}{\delta \bar{\xi}_{\mu}^{a}}\right) \bar{L}_{\mu}^{a}-\left(\phi^{\star a}+\frac{\delta \Psi}{\delta \phi^{a}}\right) M^{a}
\end{aligned}
$$




$$
\begin{aligned}
& -\left(\bar{\phi}^{\star a}+\frac{\delta \Psi}{\delta \bar{\phi}^{a}}\right) \bar{M}^{a}+\left(-e^{\star a}-\frac{\delta \Psi}{\delta e^{a}}\right) N^{a} \\
& +A_{\mu}^{a \star}\left(D^{\mu} C^{a}\right)^{a}-\frac{\bar{C}^{a \star}}{2} f^{a b c} C^{b} \xi_{\mu}^{c}+C^{\star a} b^{a} \\
& +\xi_{\mu}^{\star a}\left[\left(D_{\mu} \phi\right)^{a}+f^{a b c} C^{b} \xi_{\mu}^{c}\right]+\phi^{\star a} f^{a b c} C^{b} \xi_{\mu}^{c} .
\end{aligned}
$$

Integrating out the ghost fields associated with shift symmetry, we obtain

$$
\begin{aligned}
A_{\mu}^{a \star} & =-\frac{\delta \Psi}{\delta A^{\mu a}}, \\
\bar{C}^{\star a} & =-\frac{\delta \Psi}{\delta C^{a}}, \\
C^{\star a} & =-\frac{\delta \Psi}{\delta \bar{C}^{a}}, \\
b^{\star a} & =-\frac{\delta \Psi}{\delta b^{a}}, \\
\xi_{\mu}^{\star a} & =-\frac{\delta \Psi}{\delta \xi_{\mu}^{\star a}}, \\
\bar{\xi}_{\mu}^{\star a} & =-\frac{\delta \Psi}{\delta \bar{\xi}_{\mu}^{\star a}}, \\
\phi^{\star a} & =-\frac{\delta \Psi}{\delta \phi^{a}}, \\
\bar{\phi}^{\star a} & =-\frac{\delta \Psi}{\delta \bar{\phi}^{a}}, \\
e^{\star a} & =-\frac{\delta \Psi}{\delta e^{a}} .
\end{aligned}
$$

For a particular choice of gauge-fixing fermion $\Psi$ given in (4), anti-ghost fields get following identifications:

$$
\begin{aligned}
A_{\mu}^{a \star} & =\partial_{\mu} \bar{C}^{a}, \\
\bar{C}^{a \star} & =0, \\
C^{a \star} & =-\partial_{\mu} A^{\mu a}, \\
b^{a \star} & =0, \\
\xi_{\mu}^{\star a} & =\partial_{\mu} \bar{\phi}^{a}, \\
\bar{\xi}_{\mu}^{\star a} & =-\partial^{\nu} B_{\mu \nu}^{a}-\partial_{\mu} e^{a}, \\
\phi^{\star a} & =0, \\
\bar{\phi}^{\star a} & =-\partial^{\mu} \xi_{\mu}^{a}, \\
e^{\star a} & =\omega^{a}+\partial^{\mu} \bar{\xi}_{\mu}^{a} .
\end{aligned}
$$

It is obvious to see that, with these anti-ghost fields, the expression (15) changes to the original Lagrangian density of the BF model in Landau gauge.

\section{Extended BRST Invariant Superspace Description}

In this section, the Lagrangian density of BF model which is invariant under the extended BRST transformations only is described in a superspace $\left(x_{\mu}, \theta\right)$, where $\theta$ is a Grassmann coordinate and $x_{\mu}$ is the four-dimensional spect-time coordinates. In order to give superspace description for the extended BRST invariant theory, we first define superfields of the form

$$
\begin{aligned}
& A_{\mu}^{a}(x, \theta)=A_{\mu}^{a}+\theta \psi_{\mu}^{a}, \\
& \widetilde{A}_{\mu}^{a}(x, \theta)=\widetilde{A}_{\mu}^{a}+\theta\left[\psi_{\mu}-\left(D_{\mu}-\widetilde{D}_{\mu}\right)(C-\widetilde{C})\right]^{a}, \\
& \chi^{a}(x, \theta)=C^{a}+\theta \epsilon^{a}, \\
& \widetilde{\chi}^{a}(x, \theta)=\widetilde{C}^{a}+\theta\left[\epsilon^{a}-\frac{1}{2} f^{a b c}\left(C^{b}-\widetilde{C}^{b}\right)\left(\xi_{\mu}^{c}-\widetilde{\xi}_{\mu}^{c}\right)\right], \\
& \bar{\chi}^{a}(x, \theta)=\bar{C}^{a}+\theta \bar{\epsilon}^{a}, \\
& \widetilde{\chi^{a}}(x, \theta)=\widetilde{\bar{C}}^{a}+\theta\left[\bar{\epsilon}^{a}-(b-\widetilde{b})^{a}\right], \\
& b^{a}(x, \theta)=b^{a}+\theta \chi^{a}, \\
& \widetilde{b}^{a}(x, \theta)=\widetilde{b}^{a}+\theta \chi^{a} \\
& \xi_{\mu}^{a}(x, \theta)=\xi_{\mu}^{a}+\theta L_{\mu}^{a}, \\
& \widetilde{\xi}_{\mu}^{a}(x, \theta)=\widetilde{\xi}_{\mu}^{a}+\theta\left[L_{\mu}^{a}-\left[\left(D_{\mu}-\widetilde{D}_{\mu}\right)(\phi-\widetilde{\phi})^{a}\right.\right. \\
& \left.\left.+f^{a b c}\left(C^{b}-\widetilde{C}^{b}\right)\left(\xi_{\mu}^{c}-\widetilde{\xi}_{\mu}^{c}\right)\right]\right] \\
& \bar{\xi}_{\mu}^{a}(x, \theta)=\bar{\xi}_{\mu}^{a}+\theta \bar{L}_{\mu}^{a}, \\
& {\widetilde{\bar{\xi}_{\mu}^{a}}}_{\mu}(x, \theta)=\widetilde{\bar{\xi}_{\mu}^{a}}+\theta\left[\bar{L}_{\mu}^{a}-\left(h_{\mu}^{a}-\widetilde{h}_{\mu}^{a}\right)\right], \\
& \phi^{a}(x, \theta)=\phi^{a}+\theta M^{a} \text {, } \\
& \widetilde{\phi}^{a}(x, \theta)=\widetilde{\phi}^{a}+\theta\left[M^{a}-f^{a b c}\left(C^{b}-\widetilde{C}^{b}\right)\left(\phi^{c}-\widetilde{\phi}^{c}\right)\right], \\
& \bar{\phi}^{a}(x, \theta)=\bar{\phi}^{a}+\theta \bar{M}^{a} \text {, } \\
& {\widetilde{\phi^{a}}}^{a}(x, \theta)=\widetilde{\bar{\phi}^{a}}+\theta\left[\bar{M}^{a}-\left(\omega^{a}-\widetilde{\omega}^{a}\right)\right], \\
& e^{a}(x, \theta)=e^{a}+\theta N^{a}, \\
& \widetilde{e}^{a}(x, \theta)=\widetilde{e}^{a}+\theta\left[N^{a}-\lambda^{a}+\widetilde{\lambda}^{a}\right] .
\end{aligned}
$$

The super-antifields in superspace are defined as follows:

$$
\begin{aligned}
& \widetilde{A}_{\mu}^{\star a}(x, \theta)=A_{\mu}^{\star a}-\theta \zeta_{\mu}^{a}, \\
& \tilde{\chi}^{\star a}(x, \theta)=C^{\star a}-\theta \sigma^{a}, \\
& \tilde{\bar{\chi}}^{\star a}(x, \theta)=\bar{C}^{\star a}-\theta \bar{\sigma}^{a},
\end{aligned}
$$




$$
\begin{aligned}
& \widetilde{b}^{\star a}(x, \theta)=b^{\star a}-\theta \omega^{a}, \\
& \widetilde{\xi}_{\mu}^{\star a}(x, \theta)=\xi_{\mu}^{\star a}-\theta \kappa^{a}, \\
& \widetilde{\bar{\xi}_{\mu}^{\star a}}(x, \theta)=\bar{\xi}_{\mu}^{\star a}-\theta \bar{\kappa}^{a}, \\
& \widetilde{\phi}^{\star a}(x, \theta)=\phi^{\star a}-\theta v^{a}, \\
& \widetilde{\bar{\phi}^{\star a}}(x, \theta)=\bar{\phi}^{\star a}-\theta \bar{v}^{a}, \\
& \widetilde{e}^{\star a}(x, \theta)=e^{\star a}-\theta \tau^{a} .
\end{aligned}
$$

From the above expressions of superfields and superantifields, we calculate

$$
\begin{aligned}
& \frac{\delta\left(\widetilde{A}_{\mu}^{a \star} \widetilde{A}^{a \mu}\right)}{\delta \theta}=-A_{\mu}^{a \star}\left[\psi^{a \mu}-\left(D^{\mu}-\widetilde{D}^{\mu}\right)(C-\widetilde{C})^{a}\right] \\
& -\zeta_{\mu}^{a} \widetilde{A}^{a \mu} \\
& \frac{\delta\left(\widetilde{\widetilde{\chi}}^{a \star} \tilde{\chi}^{a}\right)}{\delta \theta}=\bar{C}^{a \star}\left[\epsilon^{a}-\frac{1}{2} f^{a b c}\left(C^{b}-\widetilde{C}^{b}\right)\left(\xi_{\mu}^{c}-\widetilde{\xi}_{\mu}^{c}\right)\right] \\
& -\bar{\sigma}^{a} \widetilde{C}^{a} \\
& \frac{\delta\left(\widetilde{\bar{\chi}}^{a} \widetilde{\chi}^{a \star}\right)}{\delta \theta}=-\sigma^{a} \widetilde{\bar{C}}^{a}+C^{a \star}\left[\bar{\epsilon}^{a}-\left(b^{a}-\widetilde{b}^{a}\right)\right], \\
& \frac{\delta\left(\widetilde{b}^{a \star} \widetilde{b}^{a}\right)}{\delta \theta}=-b^{a \star} \chi^{a}-\omega^{a} \widetilde{b}^{a}, \\
& \frac{\delta\left(\widetilde{\xi}_{\mu}^{a \star} \widetilde{\xi}^{a \mu}\right)}{\delta \theta}=\xi_{\mu}^{a \star}\left[L_{\mu}^{a}-\left[\left(D^{\mu}-\widetilde{D}^{\mu}\right)(\phi-\widetilde{\phi})^{a}\right.\right. \\
& \left.\left.+f^{a b c}\left(C^{b}-\widetilde{C}^{b}\right)\left(\xi_{\mu}^{c}-\widetilde{\xi}_{\mu}^{c}\right)\right]\right]-\kappa^{a} \widetilde{\xi}_{\mu}^{a} \\
& \frac{\delta\left(\widetilde{\left.{\widetilde{\xi_{\mu}^{a \star}}}^{\bar{\xi}^{a \mu}}\right)}\right.}{\delta \theta}=\bar{\xi}_{\mu}^{a \star}\left[L^{\mu a}-h^{\mu a}+\widetilde{h}^{\mu a}\right]-\bar{\kappa}^{a{\widetilde{\xi^{a \mu}}}}, \\
& \frac{\delta\left(\widetilde{\phi}^{a \star} \widetilde{\phi}^{a}\right)}{\delta \theta}=-\widetilde{\phi}^{a} v^{a}-\phi^{a \star}\left[M^{a}-f^{a b c}\left(C^{b}-\widetilde{C}^{b}\right)\left(\phi^{c}\right.\right. \\
& \left.-\widetilde{\phi}^{c}\right) \text {, } \\
& \frac{\delta\left(\widetilde{\widetilde{\phi}^{a \star}} \widetilde{\phi^{a}}\right)}{\delta \theta}=-\widetilde{\bar{\phi}}^{a} \bar{v}^{a}-\bar{\phi}^{a \star}\left[\bar{M}^{a}-\omega^{a}+\widetilde{\omega}^{a}\right], \\
& \frac{\delta\left(\widetilde{e}^{a \star} \widetilde{e}^{a}\right)}{\delta \theta}=-e^{\star}\left[N^{a}-\lambda^{a}+\widetilde{\lambda}^{a}\right]-\widetilde{e}^{a} \tau^{a} .
\end{aligned}
$$

Adding all the equations of (20) side by side, we get

$$
\begin{aligned}
& \frac{\delta}{\delta \theta}\left({\widetilde{A_{\mu}}}_{\mu}^{\star \star} \widetilde{A}^{a \mu}+\widetilde{\bar{\chi}^{a \star}} \widetilde{\chi}^{a}+\widetilde{\bar{\chi}^{a}} \widetilde{\chi}^{a \star}+\widetilde{b}^{a \star} \widetilde{b}^{a}+\widetilde{\xi}_{\mu}^{a \star} \widetilde{\xi}^{a \mu}\right. \\
& \left.+\widetilde{\bar{\xi}_{\mu}^{a \star}} \widetilde{\bar{\xi}^{a \mu}}+\widetilde{\phi}^{a \star} \widetilde{\phi}^{a}+\widetilde{\bar{\phi}^{a \star}} \widetilde{\bar{\phi}}^{a}+\widetilde{e}^{a \star} \widetilde{e}^{a}\right)=-\zeta^{a \mu} \widetilde{A}_{\mu}^{a}
\end{aligned}
$$

$$
\begin{aligned}
& -A_{\mu}^{a \star}\left[\psi^{a \mu}-\left(D^{\mu}-\widetilde{D}^{\mu}\right)(C-\widetilde{C})^{a}\right]-\bar{\sigma}^{a} \widetilde{C}^{a} \\
& +\bar{C}^{a \star}\left[\epsilon^{a}-\frac{1}{2} f^{a b c}\left(C^{b}-\widetilde{C}^{b}\right)\left(\xi_{\mu}^{a}-\widetilde{\xi}_{\mu}^{a}\right)\right]-\sigma^{a} \widetilde{\bar{C}}^{a} \\
& +C^{a \star}\left[\bar{\epsilon}^{a}-\left(b^{a}-\widetilde{b}^{a}\right)\right]-v^{a} \widetilde{\phi}^{a}-\phi^{\star a}\left[M^{a}\right. \\
& \left.-f^{a b c}\left(C^{b}-\widetilde{C}^{b}\right)\left(\phi^{c}-\widetilde{\phi}^{c}\right)\right]-\bar{v}^{a} \widetilde{\bar{\phi}}^{a}-\bar{\phi}^{\star a}\left(\bar{M}^{a}\right. \\
& \left.-\omega^{a}+\widetilde{\omega}^{a}\right)-\tau^{a} \widetilde{e}^{a}-e^{\star}\left[N^{a}-\lambda^{a}+\widetilde{\lambda}^{a}\right]-\omega^{a} \widetilde{b}^{a} \\
& -b^{\star a} \chi^{a}-\kappa^{a} \widetilde{\xi}_{\mu}^{a}-\bar{\kappa}^{a}{\widetilde{\xi_{\mu}^{a}}}_{\mu}+\xi_{\mu}^{\star a}\left(L_{\mu}^{a}\right. \\
& -\left[\left(D_{\mu}-\widetilde{D}_{\mu}\right)(\phi-\widetilde{\phi})^{a}\right. \\
& \left.\left.+f^{a b c}\left(C^{b}-\widetilde{C}^{b}\right)\left(\xi_{\mu}^{c}-\widetilde{\xi}_{\mu}^{c}\right)\right]\right)+\bar{\xi}_{\mu}^{\star a}\left[\bar{L}_{\mu}^{a}-\left(h_{\mu}^{a}\right.\right. \\
& \left.\left.-\widetilde{h}_{\mu}^{a}\right)\right],
\end{aligned}
$$

which is nothing but the gauge-fixed Lagrangian density for shift symmetry $\widetilde{\mathscr{L}}_{g f+g h}$ given in (12). Now, one can define the general super-gauge-fixing fermion in superspace as follows:

$$
\Phi(x, \theta)=\Psi(x)+\theta(s \Psi)
$$

which can further be expressed as

$$
\begin{aligned}
& \Phi(x, \theta)=\Psi(x)+\theta\left[-\frac{\delta \Psi}{\delta A_{\mu}^{a}} \psi_{\mu}^{a}+\frac{\delta \Psi}{\delta C^{a}} \epsilon^{a}+\frac{\delta \Psi}{\delta \bar{C}^{a}} \bar{\epsilon}^{a}\right. \\
& -\frac{\delta \Psi}{\delta b^{a}} \chi^{a}-\frac{\delta \Psi}{\delta \xi_{\mu}^{a}} L_{\mu}^{a}-\frac{\delta \Psi}{\delta \bar{\xi}_{\mu}^{a}} \bar{L}_{\mu}^{a}-\frac{\delta \Psi}{\delta \phi^{a}} M^{a}-\frac{\delta \Psi}{\delta \bar{\phi}^{a}} \bar{M}^{a} \\
& \left.-\frac{\delta \Psi}{\delta e^{a}} N^{a}\right] .
\end{aligned}
$$

From this, the original gauge-fixing Lagrangian density can be defined as the left derivation of super-gauge-fixing fermion with respect to $\theta$ as $[\delta \Phi(x, \theta) / \delta \theta]$.

Hence, the complete effective action for the BF model in general gauge in the superspace is now given by

$$
\begin{aligned}
\mathscr{L}_{\mathrm{eff}} & =\mathscr{L}_{0}+\frac{\delta}{\delta \theta}\left[\widetilde{A}_{\mu}^{a \star} \widetilde{A}^{a \mu}+\widetilde{\bar{\chi}}^{a \star} \widetilde{\chi}^{a}+\widetilde{\bar{\chi}^{a}} \widetilde{\chi}^{a \star}+\widetilde{b}^{a \star} \widetilde{b}^{a}\right. \\
& \left.+\widetilde{\chi}_{\mu}^{a \star} \widetilde{\chi}^{a \mu}+\widetilde{\bar{\chi}_{\mu}^{a \star}} \widetilde{\bar{\chi}}^{a \mu}+\widetilde{\phi}^{a \star} \widetilde{\phi}^{a}+\widetilde{\bar{\phi}^{a \star}} \widetilde{\bar{\phi}^{a}}+\widetilde{e}^{a \star} \widetilde{e}^{a}+\Phi\right] .
\end{aligned}
$$

Next, we will study the extended anti-BRST symmetry for BF model. 


\section{Extended Anti-BRST Lagrangian Density}

In this section, we construct the extended anti-BRST transformation under which the shifted Lagrangian density for $\mathrm{BF}$ model remains invariant as follows:

$$
\begin{aligned}
& \bar{s} A_{\mu}^{a}=A_{\mu}^{a \star}+\left(D_{\mu}-\widetilde{D}_{\mu}\right)(\bar{C}-\widetilde{\bar{C}})^{a}, \\
& \bar{s} \widetilde{A}_{\mu}^{a}=A_{\mu}^{a \star}, \\
& \bar{s} C^{a}=C^{a \star}-\frac{1}{2} f^{a b c}\left(C^{b}-\widetilde{C}^{b}\right)\left(\xi_{\mu}^{c}-\widetilde{\xi}_{\mu}^{c}\right), \\
& \bar{s} \widetilde{C}^{a}=C^{a \star}, \\
& \bar{s} \bar{C}^{a}=\bar{C}^{a \star}-\left(b^{a}-\widetilde{b}^{a}\right), \\
& \bar{s} \widetilde{\bar{C}^{a}}=\overline{C^{a \star}}, \\
& \bar{s} b^{a}=b^{a \star}+\chi^{a}, \\
& \bar{s} \widetilde{b}^{a}=b^{a \star}, \\
& \bar{s} \xi_{\mu}^{a}=\xi_{\mu}^{a \star}-\left[\left(D_{\mu}-\widetilde{D}_{\mu}\right)\left(\phi^{a}-\widetilde{\phi}^{a}\right)\right. \\
& \left.+f^{a b c}\left(C^{b}-\widetilde{C}^{b}\right)\left(\xi_{\mu}^{c}-\widetilde{\xi}_{\mu}^{c}\right)\right], \\
& \bar{s} \widetilde{\xi}_{\mu}^{a}=\xi_{\mu}^{a \star}, \\
& \bar{s} \bar{\xi}_{\mu}^{a}=\bar{\xi}_{\mu}^{a \star}-h_{\mu}^{a}+\widetilde{h}_{\mu}^{a} \text {, } \\
& \overline{\bar{s}} \widetilde{\bar{\xi}}_{\mu}^{a}=\bar{\xi}_{\mu}^{a \star}, \\
& \bar{s} \phi^{a}=\phi^{a \star}-f^{a b c}\left(C^{b}-\widetilde{C}^{b}\right)\left(\phi^{c}-\widetilde{\phi}^{c}\right), \\
& \bar{s} \widetilde{\phi}^{a}=\phi^{a \star}, \\
& \bar{s} \bar{\phi}^{a}=\bar{\phi}^{a \star}-\omega^{a}+\widetilde{\omega}^{a}, \\
& \widetilde{\bar{s} \phi}=\bar{\phi}^{a \star}, \\
& \bar{s} e^{a}=e^{a \star}-\left(\lambda^{a}-\tilde{\lambda}^{a}\right), \\
& \bar{s} \widetilde{e}^{a}=e^{a \star} .
\end{aligned}
$$

The ghost fields associated with the shift symmetry transform under extended anti-BRST symmetry as

$$
\begin{aligned}
\bar{s} \psi_{\mu}^{a} & =\zeta_{\mu}^{a}, \\
\bar{s} \epsilon^{a} & =\sigma^{a}, \\
\bar{s} \bar{\epsilon}^{a} & =\bar{\sigma}^{a}, \\
\bar{s} \chi^{a} & =\Phi^{a}, \\
\bar{s} L_{\mu}^{a} & =\kappa^{a}, \\
\bar{s} \bar{L}_{\mu}^{a} & =\bar{\kappa}^{a},
\end{aligned}
$$

$$
\begin{aligned}
\bar{s} M_{\mu}^{a} & =v^{a}, \\
\bar{s} M_{\mu}^{a} & =\bar{v}^{a}, \\
\bar{s} N_{\mu}^{a} & =\tau^{a} .
\end{aligned}
$$

The nilpotency of above transformations demands the auxiliary and antighost fields associated with the shift symmetry transform as

$$
\begin{aligned}
\bar{s} \zeta_{\mu}^{a} & =0, \\
\bar{s} A_{\mu}^{a \star} & =0, \\
\bar{s} \sigma^{a} & =0, \\
\bar{s} C^{a \star} & =0, \\
\bar{s} \bar{\sigma}^{a} & =0, \\
\bar{s} \bar{C}^{a \star} & =0, \\
\bar{s} \omega^{a} & =0, \\
\bar{s} b^{a \star} & =0, \\
\bar{s} \kappa^{a} & =0, \\
\bar{s} \xi_{\mu}^{a \star} & =0, \\
\bar{s} \bar{\kappa}^{a} & =0, \\
\bar{s} \bar{\xi}_{\mu}^{a \star} & =0, \\
\bar{s} v^{a} & =0, \\
\bar{s} \phi^{a \star} & =0, \\
\bar{s} \bar{v}^{a} & =0, \\
\bar{s} \bar{s}^{a \star} & =0, \\
\bar{s} \tau^{a} & =0, \\
\bar{s} e^{a \star} & =0 .
\end{aligned}
$$

The gauge-fixing and ghost parts of the effective Lagrangian density are anti-BRST-exact also so it can be expressed as the anti-BRST variation of this gauge-fixing fermion $(\bar{\Psi})$.

\section{Extended BRST and Anti-BRST Invariant Superspace}

The extended BRST and anti-BRST invariant Lagrangian density for BF model can be written in superspace with the help of two additional Grassmannian coordinates $\theta$ and $\bar{\theta}$. 
Requiring the field strength to vanish along unphysical directions $\theta$ and $\bar{\theta}$ direction, we obtain the following superfields:

$$
\begin{aligned}
& \mathbf{A}_{\mu}^{a}(x, \theta, \bar{\theta})=A_{\mu}^{a}(x)+\theta \psi_{\mu}^{a}+\bar{\theta}\left[A_{\mu}^{a \star}+\left(D_{\mu}-\widetilde{D}_{\mu}\right)\right. \\
& \left.\cdot(\bar{C}-\widetilde{\bar{C}})^{a}\right]+\theta \bar{\theta} \zeta_{\mu}^{a}, \\
& \widetilde{\mathbf{A}}_{\mu}^{a}(x, \theta, \bar{\theta})=\widetilde{A}_{\mu}^{a}(x)+\theta\left[\psi_{\mu}^{a}-\left(D_{\mu}-\widetilde{D}_{\mu}\right)(C-\widetilde{C})^{a}\right] \\
& +\bar{\theta} A_{\mu}^{a \star}+\theta \bar{\theta} \zeta_{\mu}^{a}, \\
& \mathbf{C}^{a}(x, \theta, \bar{\theta})=C^{a}(x)+\theta \epsilon^{a}+\bar{\theta}\left[C^{a \star}-\frac{1}{2} f^{a b c}\left(C^{b}\right.\right. \\
& \left.\left.-\widetilde{C}^{b}\right)\left(\xi_{\mu}^{c}-\widetilde{\xi}_{\mu}^{c}\right)\right]+\theta \bar{\theta} \sigma^{a}, \\
& \widetilde{\mathbf{C}}^{a}(x, \theta, \bar{\theta})=\widetilde{C}^{a}(x)+\theta\left[\epsilon^{a}-\frac{1}{2} f^{a b c}\left(C^{b}-\widetilde{C}^{b}\right)\left(\xi_{\mu}^{c}\right.\right. \\
& \left.\left.-\widetilde{\xi}_{\mu}^{c}\right)\right]+\bar{\theta} C^{\star a}+\theta \bar{\theta} \sigma^{a}, \\
& \overline{\mathbf{C}}^{a}(x, \theta, \bar{\theta})=\bar{C}^{a}(x)+\theta \bar{\epsilon}^{a}+\bar{\theta}\left[\bar{C}^{\star a}-(b-\widetilde{b})^{a}\right] \\
& +\theta \bar{\theta} \bar{\sigma}^{a}, \\
& \widetilde{\overline{\mathbf{C}}^{a}}(x, \theta, \bar{\theta})=\widetilde{\overline{\bar{C}}^{a}}(x)+\theta\left[\bar{\epsilon}^{a}-(b-\widetilde{b})^{a}\right]+\bar{\theta} \overline{\bar{C}}^{\star a} \\
& +\theta \bar{\theta} \bar{\sigma}^{a} \\
& \mathbf{b}^{a}(x, \theta, \bar{\theta})=b^{a}(x)+\theta \chi^{a}+\bar{\theta}\left(b^{\star a}+\chi^{a}\right)+\theta \bar{\theta} \omega^{a}, \\
& \widetilde{\mathbf{b}}^{a}(x, \theta, \bar{\theta})=\widetilde{b}^{a}(x)+\theta \chi^{a}+\bar{\theta} b^{\star a}+\theta \bar{\theta} \omega^{a}, \\
& \xi_{\mu}^{a}(x, \theta, \bar{\theta})=\xi_{\mu}^{a}(x)+\theta L_{\mu}^{a}+\bar{\theta}\left(\xi_{\mu}^{a \star}\right. \\
& \text { - }\left[\left(D_{\mu}-\widetilde{D}_{\mu}\right)\left(\phi^{a}-\widetilde{\phi}^{a}\right)\right. \\
& \left.\left.+f^{a b c}\left(C^{b}-\widetilde{C}^{b}\right)\left(\xi_{\mu}^{c}-\widetilde{\xi}_{\mu}^{c}\right)\right]\right)+\theta \bar{\theta} \kappa^{a}, \\
& \widetilde{\xi}_{\mu}^{a}(x, \theta, \bar{\theta})=\widetilde{\xi}_{\mu}^{a}(x)+\theta\left[L_{\mu}^{a}-\left(D_{\mu}-\widetilde{D}_{\mu}\right)(\phi-\widetilde{\phi})^{a}\right. \\
& \left.+f^{a b c}\left(C^{b}-\widetilde{C}^{b}\right)\left(\xi_{\mu}^{c}-\widetilde{\xi}_{\mu}^{c}\right)\right]+\bar{\theta} \xi_{\mu}^{a \star}+\theta \bar{\theta} \kappa^{a}, \\
& \bar{\xi}_{\mu}^{a}(x, \theta, \bar{\theta})=\bar{\xi}_{\mu}^{a}(x)+\theta \bar{L}_{\mu}^{a}+\bar{\theta}\left(\bar{\xi}_{\mu}^{\star a}-h_{\mu}^{a}+\widetilde{h}_{\mu}^{a}\right) \\
& +\theta \bar{\theta} \bar{\kappa}^{a}, \\
& {\widetilde{\xi^{a}}}_{\mu}(x, \theta, \bar{\theta})={\widetilde{\xi_{\mu}^{a}}}_{\mu}(x)+\theta\left(\bar{L}_{\mu}^{a}-h_{\mu}^{a}+\widetilde{h}_{\mu}^{a}\right)+\bar{\theta}_{\mu}^{\star a} \\
& +\theta \bar{\theta} \bar{\kappa}^{a}, \\
& \phi^{a}(x, \theta, \bar{\theta})=\phi^{a}(x)+\theta M^{a}+\bar{\theta}\left(\phi^{a \star}-f^{a b c}\left(C^{b}\right.\right. \\
& \left.\left.-\widetilde{C}^{b}\right)\left(\phi^{c}-\widetilde{\phi}^{c}\right)\right)+\theta \bar{\theta} v^{a},
\end{aligned}
$$

$$
\begin{aligned}
& \widetilde{\phi}^{a}(x, \theta, \bar{\theta})=\widetilde{\phi}^{a}(x)+\theta\left(M^{a}-f^{a b c}\left(C^{b}-\widetilde{C}^{b}\right)\left(\phi^{c}\right.\right. \\
& \left.\left.-\widetilde{\phi}^{c}\right)\right)+\bar{\theta} \phi^{\star a}+\theta \bar{\theta} v^{a}, \\
& \bar{\phi}^{a}(x, \theta, \bar{\theta})=\bar{\phi}^{a}(x)+\theta \bar{M}^{a}+\bar{\theta}\left(\bar{\phi}^{\star a}-\omega^{a}+\widetilde{\omega}^{a}\right) \\
& \quad+\theta \bar{\theta} \bar{v}^{a}, \\
& \widetilde{\bar{\phi}}^{a}(x, \theta, \bar{\theta})=\widetilde{\bar{\phi}}^{a}(x)+\theta\left(\bar{M}^{a}-\omega^{a}+\widetilde{\omega}^{a}\right)+\overline{\theta \phi}^{\star a} \\
& \quad+\theta \bar{\theta} \bar{v}^{a}, \\
& \mathbf{e}^{a}(x, \theta, \bar{\theta})=e^{a}(x)+\theta N^{a}+\bar{\theta}\left(e^{\star a}-\lambda^{a}+\widetilde{\lambda}^{a}\right) \\
& \quad+\theta \bar{\theta} \tau^{a}, \\
& \widetilde{\mathbf{e}}^{a}(x, \theta, \bar{\theta})=\widetilde{e}^{a}(x)+\theta\left(N^{a}-\lambda^{a}+\widetilde{\lambda}^{a}\right)+\bar{\theta} e^{\star a} \\
& \quad+\theta \bar{\theta} \tau^{a} .
\end{aligned}
$$

With these expressions of superfields, we can calculate

$$
\begin{aligned}
& -\frac{1}{2} \frac{\partial}{\partial \bar{\theta}} \frac{\partial}{\partial \theta}\left(\widetilde{\mathbf{A}}_{\mu}^{a} \widetilde{\mathbf{A}}^{\mu a}+\widetilde{\chi}^{a} \widetilde{\bar{\chi}}^{a}+\widetilde{\mathbf{b}}^{a} \widetilde{\mathbf{b}}^{a}+\widetilde{\xi}_{\mu}^{a} \widetilde{\xi}^{\mu a}+\widetilde{\bar{\xi}}_{\mu}^{a} \widetilde{\bar{\xi}}^{\mu a}\right. \\
& \left.+\widetilde{\phi}^{a} \widetilde{\phi}^{a}+\widetilde{\bar{\phi}^{a}} \widetilde{\bar{\phi}}^{a}+\widetilde{\mathbf{e}}^{a} \widetilde{\mathbf{e}}^{a}\right)=-\zeta^{a \mu} \widetilde{A}_{\mu}^{a}-A_{\mu}^{a \star}\left[\psi^{a \mu}\right. \\
& \left.-\left(D^{\mu}-\widetilde{D}^{\mu}\right)(C-\widetilde{C})^{a}\right]-\bar{\sigma}^{a} \widetilde{C}^{a}+\bar{C}^{a \star}\left[\epsilon^{a}-\frac{1}{2}\right. \\
& \left.\cdot f^{a b c}\left(C^{b}-\widetilde{C}^{b}\right)\left(\xi_{\mu}^{a}-\widetilde{\xi}_{\mu}^{a}\right)\right]-\sigma^{a} \widetilde{C}^{a}+C^{a \star}\left[\bar{\epsilon}^{a}\right. \\
& \left.-\left(b^{a}-\widetilde{b}^{a}\right)\right]-v^{a} \widetilde{\phi}^{a}-\phi^{\star a}\left[M^{a}-f^{a b c}\left(C^{b}-\widetilde{C}^{b}\right)\right. \\
& \left.-\left(\phi^{c}-\widetilde{\phi}^{c}\right)\right]-\bar{v}^{a} \widetilde{\bar{\phi}}^{a}-\bar{\phi}^{\star a}\left(\bar{M}^{a}-\omega^{a}+\widetilde{\omega}^{a}\right)-\tau^{a} \widetilde{e}^{a} \\
& -e^{\star}\left[N^{a}-\lambda^{a}+\widetilde{\lambda}^{a}\right]-\omega^{a} \widetilde{b}^{a}-b^{\star a} \chi^{a}-\kappa^{a} \widetilde{\xi}_{\mu}^{a} \\
& -\bar{\kappa}^{a} \widetilde{\xi}_{\mu}^{a}+\xi_{\mu}^{\star a}\left(L_{\mu}^{a}-\left[\left(D_{\mu}-\widetilde{D}_{\mu}\right)(\phi-\widetilde{\phi})^{a}\right.\right. \\
& \left.\left.+f^{a b c}\left(C^{b}-\widetilde{C}^{b}\right)\left(\xi_{\mu}^{c}-\widetilde{\xi}_{\mu}^{c}\right)\right]\right)+\bar{\xi}_{\mu}^{\star a}\left[\bar{L}_{\mu}^{a}-\left(h_{\mu}^{a}\right.\right. \\
& \left.\left.-\widetilde{h}_{\mu}^{a}\right)\right],
\end{aligned}
$$

which is nothing but the gauge-fixed Lagrangian density for shift symmetry. Being the $\theta \bar{\theta}$ component of a superfield, this Lagrangian density is manifestly invariant under both the extended BRST and the anti-BRST transformations.

Now, we define the general super-gauge-fixing fermion in superspace as

$$
\Phi(x, \theta, \bar{\theta})=\Psi(x)+\theta(s \Psi)+\bar{\theta}(\bar{s} \Psi)+\theta \bar{\theta}(s \bar{s} \Psi),
$$

which yields the original gauge-fixing and ghost part of the effective Lagrangian density upon differentiation as follows: $\operatorname{Tr}[(\partial / \partial \theta)[\delta(\bar{\theta}) \Phi(x, \theta, \bar{\theta})]]$. 
Therefore, the gauge-fixed Lagrangian density corresponding to BRST and shift symmetries for BF model can now be given as

$$
\begin{aligned}
& \mathscr{L}_{g f+g h}+\widetilde{\mathscr{L}}_{g f+g h}=-\frac{1}{2} \frac{\partial}{\partial \bar{\theta}} \frac{\partial}{\partial \theta}\left(\widetilde{\mathbf{A}}_{\mu}^{a} \widetilde{\mathbf{A}}^{\mu a}+\widetilde{\chi}^{a} \widetilde{\bar{\chi}}^{a}\right. \\
& \left.+\widetilde{\mathbf{b}}^{a} \widetilde{\mathbf{b}}^{a}+\widetilde{\xi}_{\mu}^{a} \widetilde{\xi}^{\mu a}+\widetilde{\bar{\xi}_{\mu}^{a}} \widetilde{\bar{\xi}^{\mu a}}+\widetilde{\phi}^{a} \widetilde{\phi}^{a}+\widetilde{\bar{\phi}} \widetilde{\bar{\phi}^{a}}+\widetilde{\mathbf{e}}^{a} \widetilde{\mathbf{e}}^{a}\right) \\
& +\frac{\partial}{\partial \theta}[s(\bar{\theta}) \Phi(x, \theta, \bar{\theta})] .
\end{aligned}
$$

Therefore, we see that the BF model in superspace can be expressed in an elegant manner.

\section{Conclusion}

The $(3+1)$ dimensional BF model is a subject of great interest due to its topological nature and its intriguing properties. In present work, we have considered $(3+1)$ dimensional $\mathrm{BF}$ model in Landau gauge and then we have shifted the Lagrangian to obtain the extended BRST and anti-BRST invariant (including some shift symmetry) BF model in BV formulation. The antifields corresponding to each field naturally arise. Further we have provide the superfield description of $\mathrm{BF}$ model in superspace, where we show that the BV action for BF model can be written in a manifestly extended BRST invariant manner in a superspace by considering one additional Grassmann (fermionic) coordinate. However, we need two additional Grassmann coordinates to express both the extended BRST and extended anti-BRST invariant BV actions of BF model in superspace.

\section{Conflicts of Interest}

The author declares that there are no conflicts of interest regarding the publication of this paper.

\section{Acknowledgments}

The author is grateful to Dr. Sudhaker Upadhyay for his suggestions in preparation of the manuscript.

\section{References}

[1] D. Birmingham, M. Blau, M. Rakowski, and G. Thompson, “Topological field theory," Physics Reports, vol. 209, no. 4-5, pp. 129-340, 1991.

[2] C. Lucchesi, O. Piguet, and S. Sorella, "Renormalization and finiteness of topological BF theories," Nuclear Physics. B. Theoretical, Phenomenological, and Experimental High Energy Physics. Quantum Field Theory and Statistical Systems, vol. 395, no. 1-2, pp. 325-353, 1993.

[3] V. I. Ogievetsky and I. V. Polubarinov, Yadernaya fizika, vol. 4, no. 68 , p. 210.

[4] S. DESER, "Lagrangian Forms of the Dynamical Theory of Currents," Physical Review A: Atomic, Molecular and Optical Physics, vol. 187, no. 5, pp. 1931-1934, 1969.
[5] K. Hayashi, "The A1 and $\rho$ mesons from the violation of local Lorentz invariance," Physics Letters B, vol. 44, no. 6, pp. 497-501, 1973.

[6] M. Kalb and P. Ramond, "Classical direct interstring action," Physical Review D: Particles, Fields, Gravitation and Cosmology, vol. 9, no. 8, pp. 2273-2284, 1974.

[7] Y. Nambu, "III. Magnetic and electric confinement of quarks," Physics Reports, vol. 23, no. 3, pp. 250-253, 1976.

[8] D. Z. Freedman and P. K. Townsend, "Antisymmetric tensor gauge theories and non-linear $\sigma$-models," Nuclear Physics B, vol. 177, no. 2, pp. 282-296, 1981.

[9] E. Witten, "2 + 1 dimensional gravity as an exactly soluble system," Nuclear Physics B, vol. 311, no. 1, pp. 46-78, 1988.

[10] E. Guadagnini, M. Maggiore, and S. P. Sorella, "Supersymmetry of the three-dimensional Einstein-Hilbert gravity in the Landau gauge," Physics Letters B, vol. 247, no. 4, pp. 543-548, 1990.

[11] N. Maggiore and S. P. Sorella, "Perturbation theory for antisymmetric tensor fields in four dimensions," International Journal of Modern Physics A, vol. 8, no. 5, pp. 929-945, 1993.

[12] G. T. Horowitz, "Exactly soluble diffeomorphism invariant theories," Communications in Mathematical Physics, vol. 125, no. 3, pp. 417-437, 1989.

[13] G. T. Horowitz and M. Srednicki, "A quantum field theoretic description of linking numbers and their generalization," Communications in Mathematical Physics, vol. 130, no. 1, pp. 83-94, 1990.

[14] M. Blau and G. Thompson, "Topological gauge theories of antisymmetric tensor fields," Annals of Physics, vol. 205, no. 1, pp. 130-172, 1991.

[15] M. Blau and G. Thompson, "Do metric independent classical actions lead to topological field theories?" Physics Letters. B. Particle Physics, Nuclear Physics and Cosmology, vol. 255, no. 4, pp. 535-542, 1991.

[16] N. Maggiore and S. P. Sorella, "Finiteness of the topological models in the Landau gauge," Nuclear Physics B, vol. 377, no. 1-2, pp. 236-251, 1992.

[17] M. Henneaux and C. Teitelboim, Quantization of Gauge Systems, Princeton University Press, Princeton, NJ, USA, 1992.

[18] S. Weinberg, The quantum theory of fields, Vol-II: Modern applications, Cambridge University Press, Cambridge, UK, 1996.

[19] I. A. Batalin and G. A. Vilkovisky, "Gauge algebra and quantization," Physics Letters B, vol. 102, no. 1, pp. 27-31, 1981.

[20] I. A. Batalin and G. A. Vilkovisky, "Quantization of gauge theories with linearly dependent generators," Physical Review D: Particles, Fields, Gravitation and Cosmology, vol. 28, no. 10, pp. 2567-2582, 1983.

[21] J. Zinn-Justin, Renormalization of Gauge Theories, Springer, Berlin, Germany, 1975.

[22] S. Upadhyay and P. K. Panigrahi, "Quantum gauge freedom in very special relativity," Nuclear Physics B, vol. 915, pp. 168-183, 2017.

[23] S. Upadhyay, A. Reshetnyak, and B. P. Mandal, "Comments on interactions in the SUSY models," The European Physical Journal C, vol. 76, no. 7, article no. 391, 2016.

[24] S. Upadhyay, M. Oksanen, and R. Bufalo, "BRST Quantization of Unimodular Gravity," Brazilian Journal of Physics, vol. 47, no. 3, pp. 350-365, 2017. 
[25] S. Upadhyay, "Super-group field cosmology in BatalinVilkovisky formulation," International Journal of Theoretical Physics, vol. 55, no. 9, pp. 4005-4015, 2016.

[26] S. Upadhyay, " $N=1$ super-Chern-Simons theory in BatalinVilkovisky formulation," EPL (Europhysics Letters), vol. 104, Article ID 61001, 2013.

[27] S. Upadhyay, "Finite field-dependent symmetries in perturbative quantum gravity," Annals of Physics, vol. 340, pp. 110-118, 2014.

[28] S. Upadhyay, "Finite field dependent BRST transformations and its applications to gauge field theories," 2013, https://arxiv.org/ abs/1308.0982.

[29] M. Sharif and Z. Yousaf, "Stability of regular energy density in Palatini $f(R)$ gravity," The European Physical Journal C, vol. 75, p. 58, 2015.

[30] S. Upadhyay and B. P. Mandal, "Generalized BRST symmetry for arbitrary spin conformal field theory," Physics Letters. B. Particle Physics, Nuclear Physics and Cosmology, vol. 744, pp. 231-236, 2015.

[31] S. Upadhyay and B. P. Mandal, "Field dependent nilpotent symmetry for gauge theories," The European Physical Journal C, vol. 72, no. 7, article 2065, 2012.

[32] S. Upadhyay and B. P. Mandal, "Relating Gribov-Zwanziger and Yang-Mills theories in Batalin-Vilkovisky formalism," AIP Conference Proceedings, vol. 1444, p. 213, 2012.

[33] S. Upadhyay and B. P. Mandal, "Generalized BRST transformation in Abelian rank-2 antisymmetric tensor field theory," Modern Physics Letters A, vol. 25, no. 40, pp. 3347-3362, 2010.

[34] S. Upadhyay and D. Das, "ABJM theory in Batalin-Vilkovisky formulation," Physics Letters B, vol. 733, pp. 63-68, 2014.

[35] S. Upadhyay, M. K. Dwivedi, and B. P. Mandal, “The noncovariant gauges in 3-form theories," International Journal of Modern Physics A, vol. 28, no. 10, Article ID 1350033, 11 pages, 2013.

[36] S. Upadhyay and P. A. Ganai, "Finite field-dependent symmetry in the Thirring model," Progress of Theoretical and Experimental Physics, vol. 1, Article ID 063B04, 2016.

[37] C. Becchi, A. Rouet, and R. Stora, "Renormalization of the abelian Higgs-Kibble model," Communications in Mathematical Physics, vol. 42, pp. 127-162, 1975.

[38] C. Becchi, A. Rouet, and R. Stora, "Renormalization of gauge theories," Annals of Physics, vol. 98, no. 2, pp. 287-321, 1976.

[39] P. Yu. Moshin, S. Upadhyay, and R. A. Castro, "Finite BRST mapping in higher-derivative models," Brazilian Journal of Physics, vol. 47, no. 4, pp. 411-418, 2017.

[40] S. Upadhyay, "Construction of a massive ABJM theory without Higgs superfields,” Europhysics Letters, vol. 117, p. 11001, 2017.

[41] S. Upadhyay, "A mass-deformed BLG theory in GribovZwanziger framework," International Journal of Modern Physics A, vol. 31, no. 26, Article ID 1650148, 11 pages, 2016.

[42] S. Upadhyay, "Ward and Nielsen identities for ABJM theory in $N=1$ superspace," International Journal of Modern Physics A, vol. 31, Article ID 1650112, 2016.

[43] S. Upadhyay, "Ward identities and gauge flow for M-theory in $N=3$ superspace," Physical Review D: Particles, Fields, Gravitation and Cosmology, vol. 92, no. 6, Article ID 065027, 7 pages, 2015.
[44] L. R. Ribeiro, E. Passos, C. Furtado, and J. R. Nascimento, "Geometric phases modified by a Lorentz-symmetry violation background," International Journal of Modern Physics A, vol. 30, no. 14, Article ID 1550072, 2015.

[45] S. Upadhyay, "Field-dependent symmetries in FriedmannRobertson-Walker models," Annals of Physics, vol. 356, pp. 299305, 2015.

[46] S. Upadhyay, "The conformal gauge to the derivative gauge for worldsheet gravity," Physics Letters B, vol. 740, pp. 341-344, 2015.

[47] S. Upadhyay, "Nilpotent symmetries in supergroup field cosmology," Modern Physics Letters A, vol. 30, no. 18, p. 1550072, 2015.

[48] S. Upadhyay, "Generalised BRST symmetry and gaugeon formalism for perturbative quantum gravity: novel observation," Annals of Physics, vol. 344, pp. 290-300, 2014.

[49] B. P. Mandal, S. K. Rai, and S. Upadhyay, "Finite nilpotent symmetry in Batalin-Vilkovisky formalism," EPL (Europhysics Letters), vol. 105, Article ID 21001, 2014.

[50] S. Upadhyay, "Gaugeon formalism for perturbative quantum gravity," The European Physical Journal C, vol. 74, no. 2, article 2737, 2014.

[51] S. Upadhyay, "BRST symmetry and Darboux transformations in Abelian 2-form gauge theory," EPL (Europhysics Letters), vol. 103, Article ID 61002, 2013.

[52] S. Upadhyay, "Aspects of finite field-dependent symmetry in SU (2) Cho-Faddeev-Niemi decomposition," Physics Letters B, vol. 727, pp. 293-297, 2013.

[53] S. Upadhyay and B. Paul, "BRST symmetry for Regge-Teitelboim-based minisuperspacemodels," The European Physical Journal C, vol. 76, article 394, 2016.

[54] M. Faizal and S. Upadhyay, "Spontaneous breaking of the BRST symmetry in the ABJM theory," Physics Letters B, vol. 736, pp. 288-292, 2014.

[55] L. Bonora and M. Tonin, "Superfield formulation of extended BRS symmetry," Physics Letters B, vol. 98, no. 1-2, pp. 48-50, 1981.

[56] J. Alfaro and P. H. Damgaard, "Schwinger-Dyson equations as supersymmetric ward identities," Physics Letters B, vol. 222, no. 3-4, pp. 425-428, 1989.

[57] J. Alfaro, P. H. Damgaard, J. Latorre, and D. Montano, "On the BRST invariance of field deformations," Physics Letters B, vol. 233, no. 1-2, pp. 153-157, 1989.

[58] J. Alfaro and P. H. Damgaard, "Origin of antifields in the Batalin-Vilkovisky Lagrangian formalism," Nuclear Physics. B. Theoretical, Phenomenological, and Experimental High Energy Physics. Quantum Field Theory and Statistical Systems, vol. 404, no. 3, pp. 751-793, 1993.

[59] N. R. F. Braga and A. Das, "A superspace formulation of the BV action," Nuclear Physics B, vol. 442, no. 3, pp. 655-668, 1995.

[60] S. Upadhyay, M. B. Shah, and P. A. Ganai, "Lorentz violating p-form gauge theories in superspace," The European Physical Journal C, vol. 77, no. 3, 2017.

[61] S. Upadhyay, "A superspace description of FriedmannRobertson-Walker models," Progress of Theoretical and Experimental Physics, vol. 2015, no. 9, Article ID 093B06, 2015.

[62] S. Upadhyay and B. P. Mandal, "BV formulation of higher form gauge theories in a superspace," The European Physical Journal C, vol. 72, no. 7, article 2059, 2012. 
[63] S. Upadhyay, M. K. Dwivedi, and B. P. Mandal, "A superspace description of Chern-Simons theory in Batalin-Vilkovisky formulation," International Journal of Theoretical Physics, vol. 54, no. 6, pp. 2076-2086, 2015.

[64] S. Upadhyay, "Perturbative quantum gravity in Batalin-Vilkovisky formalism," Physics Letters. B. Particle Physics, Nuclear Physics and Cosmology, vol. 723, no. 4-5, pp. 470-474, 2013.

[65] P. Brax and J. Martin, "Shift symmetry and inflation in supergravity," Physical Review D: Particles, Fields, Gravitation and Cosmology, vol. 72, no. 2, Article ID 023518, 24 pages, 2005.

[66] A. Hebecker, A. K. Knochel, and T. Weigand, "A shift symmetry in the Higgs sector: experimental hints and stringy realizations," Journal of High Energy Physics, vol. 2012, no. 6, article 93, 2012. 

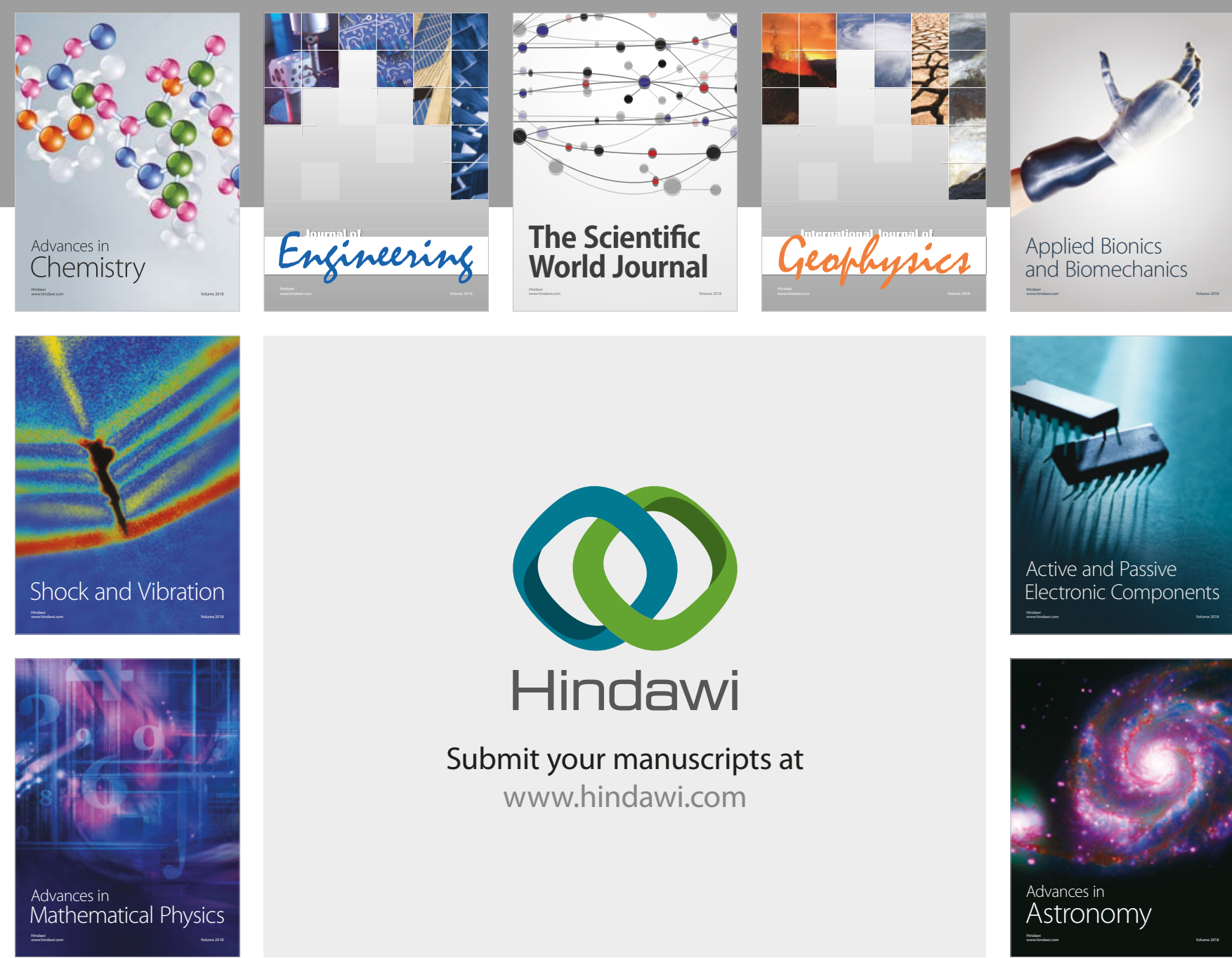

Submit your manuscripts at

www.hindawi.com

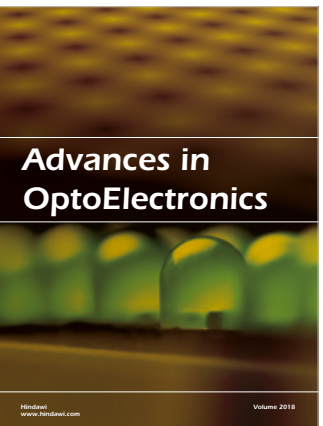

\section{Rotcting Machinery}
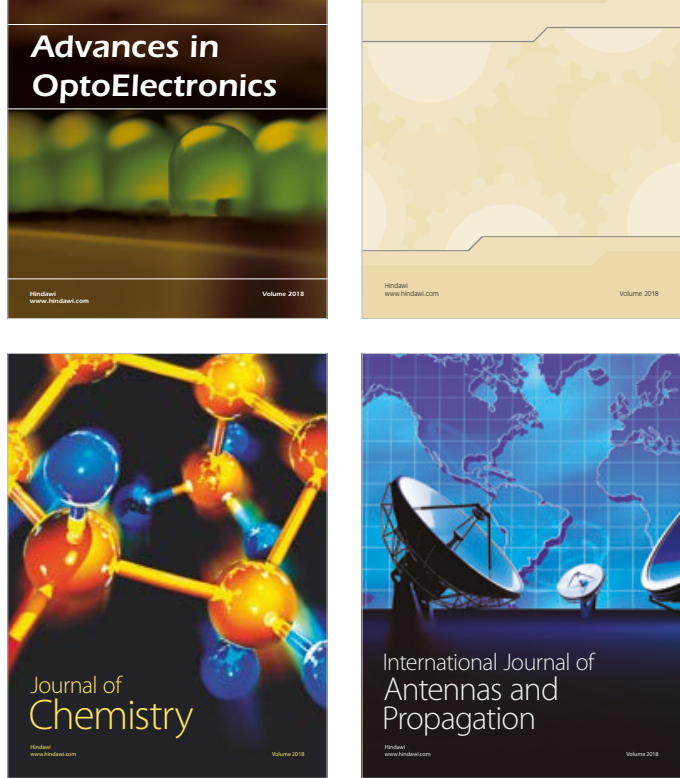

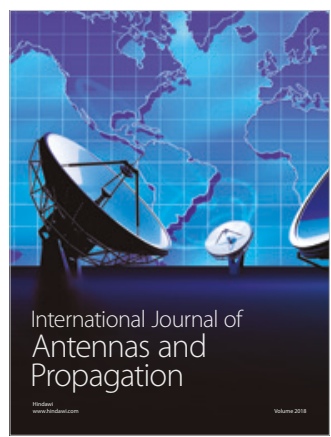

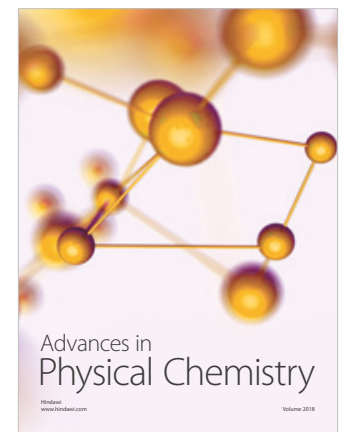

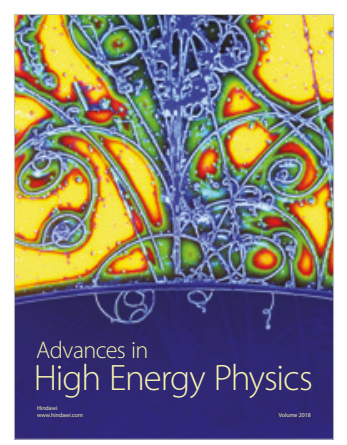

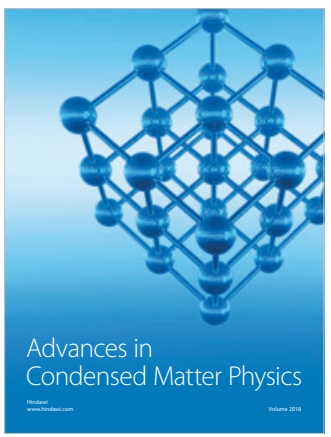

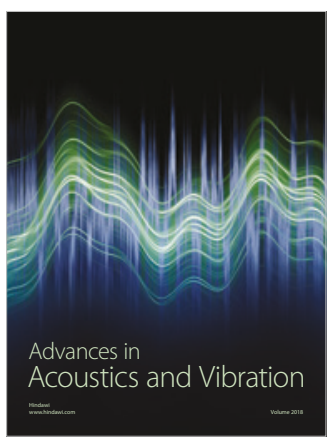

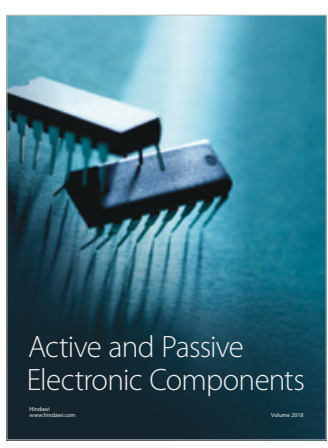
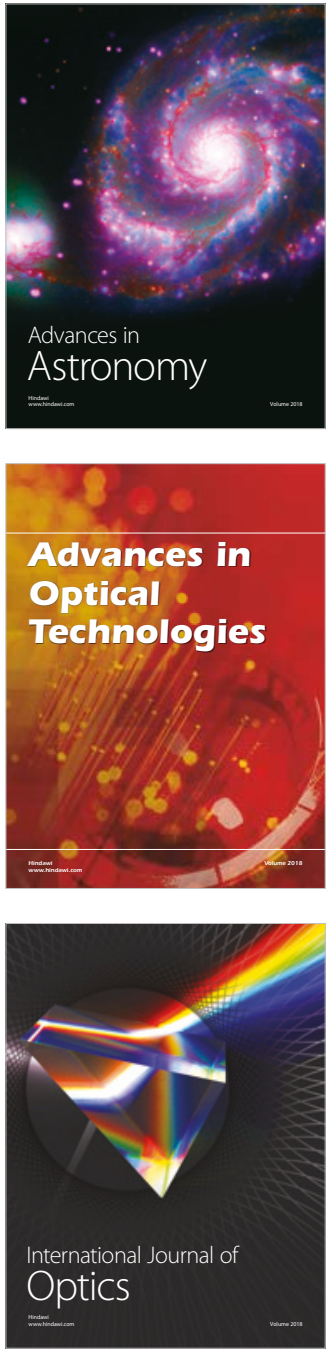